Ramos, F. \& Segovia, J. (2021). Los equinodermos de

El Salvador depositados en colecciones científicas.

Revista de Biología Tropical, 69(S1), 254-264. DOI

10.15517/rbt.v69iSuppl.1.46357

DOI 10.15517/rbt.v69iSuppl.1.46357

\title{
Los equinodermos de El Salvador depositados en colecciones científicas
}

\author{
Fernanda Ramos ${ }^{1 *}$ \\ Johanna Segovia ${ }^{2}$
}

1. Escuela de Biología, Facultad de Ciencias Naturales y Matemática, Universidad de El Salvador; ramos.fernandasv@gmail.com (*Correspondencia).

2. Universidad Francisco Gavidia; johannaseg@gmail.com; e.vsegovia@ufg.edu.sv

Recibido 30-VI-2020. Corregido 03-VIII-2020. Aceptado 16-IX-2020.

\section{ABSTRACT \\ Echinoderms from EI Salvador deposited at scientific collections}

\begin{abstract}
Introduction: The value of scientific collections lies in the fact that the records are directly linked to real physical specimens allowing us to learn the advances in taxonomy, biogeography and evolutionary history of the species. In El Salvador, the scientific collections are represented mostly by specimens of terrestrial vertebrates and, to a lesser proportion, marine invertebrates, specifically for the group of echinoderms their representation is scarce. Objective: To enlist the scientific collections in which echinoderm specimens of El Salvador are deposited, to know about the efforts made to collect these specimens and evaluate the contribution of the collections to the knowledge of the taxon in the country. Methods: Review of open databases of 18 foreign and national collections; collecting biological information, number of specimens, site, year of collection and collector. Results: 536 specimens were found in 192 records of echinoderms deposited in six scientific collections, national and foreign, classified into 40 species, one of them new for El Salvador: Ophionereis perplexa. The National Museum of Natural History (Smithsonian) and the Institute of Marine Sciences and Limnology of the University of El Salvador have the largest number of specimens (Smithsonian has the oldest specimens). Conclusions: The representation of specimens in national and foreign scientific collections is acceptable, with $67 \%$ of the total reported for El Salvador. We recommend continuation of work in shallow-waters and start of deep-water study.
\end{abstract}

Key words: collects; biodiversity; Ophionereis perplexa; records; La Union.

Las colecciones científicas son repositorios físicos conservados, catalogados y administrados por instituciones públicas u otras entidades de investigación como museos, universidades e institutos dedicados a la generación de conocimiento; en general, el reservorio de estas colecciones es documentado y difundido con el objetivo de ponerlo a disposición de la comunidad científica y la sociedad en general (Darrigan, 2012). Su valor radica en que los registros están vinculados directamente a especímenes físicos, permitiendo mejorar los avances en taxonomía, biogeografía e historia evolutiva de las especies; además de ser un medio importante de intercambio de información entre instituciones y científicos (Simmons \& Muñoz-Saba, 2005; Chicas-Batres \& González-Leiva, 2015). Estas colecciones deben preservar sus ejemplares con distintos métodos de conservación de forma que propicien análisis morfológicos y moleculares, ya que por medio de ellos es posible determinar cambios en el tiempo y 
zonas geográficas (Cristín \& Perrilliat, 2011; Darrigan, 2012).

En cuanto a la investigación con equinodermos y recolecta de estos ejemplares, en Latinoamérica poseen un origen en común con expediciones científicas provenientes de Europa y Estados Unidos de América (E.U.A) durante el siglo XIX, seguido por un periodo de investigación realizado por científicos regionales a mediados del siglo $\mathrm{XX}$, tales como María Elena Caso, Irene Bernasconi y Luiz Roberto Tommasi. Gran parte de los especímenes recolectados durante estas expediciones fueron depositados en colecciones científicas de E.U.A., Francia, Alemania y Dinamarca (Alvarado \& Solís-Marín, 2013). Actualmente, la Colección Nacional de Equinodermos "Dra. Ma. Elena Caso", depositada en el Instituto de Ciencias del Mar y Limnología de la Universidad Nacional Autónoma de México es la más extensa de América Latina (ICML-UNAM, 2019), en donde además de los ejemplares recolectados en costas mexicanas, se resguardan ejemplares de diferentes países como Belice, El Salvador, Brasil, Colombia, Costa Rica y otros (UNAM, 2013).

El Salvador posee diferentes colecciones científicas, entre las que figuran la Colección de Paleontología, Rocas y Minerales, Herbario Nacional, Colección de Malacología, Entomología, Ictiología, Herpetología, Ornitología y Mastozoología pertenecientes al Museo de Historia Natural de El Salvador (MUHNES) (Echeverría, 2013). En el Museo de la Escuela de Biología de la Universidad de El Salvador (UES) se encuentra la Colección de Referencia (Científica) y Didáctica, la primera con ejemplares de peces, anfibios, reptiles, aves y mamíferos, así como algunos taxones de invertebrados marinos (D.A. Durán, comunicación personal, 18 de agosto de 2020). En el Instituto de Ciencias del Mar y Limnología de la UES (ICMARES), se encuentra la Colección Ictiológica y la de Invertebrados Acuáticos, esta última posee 98 ejemplares de equinodermos (Segovia, Guerra, López, \& Ramos, 2016). Una característica común que poseen todas las colecciones zoológicas en el país es que los grupos mayormente representados son vertebrados terrestres y en menor cantidad invertebrados como insectos, moluscos y bivalvos. Por ello, en términos generales, en comparación a las demás colecciones, el número de ejemplares de equinodermos representados es bajo.

En este sentido, como parte de los esfuerzos por documentar los organismos marinos que habitan en el país, se han realizado expediciones científicas que han recolectado diferentes especies. Barraza y Hasbún (2005) describen que durante marzo-abril de 2001 el buque de investigación URRACÁ del Smithsonian Tropical Research Institute (STRI) tomó muestras de equinodermos en la costa salvadoreña, desde la zona intermareal hasta $400 \mathrm{~m}$ de profundidad, de estas, cinco ejemplares se depositaron en el MUHNES y el resto en colecciones extranjeras. Por otro lado, el ICMARES realizó un programa de monitoreo de arrecifes rocosos y coralinos en El Salvador entre 2012 y 2018 con mayores esfuerzos de recolección de equinodermos entre 2014-2015.

Las investigaciones con equinodermos se han enfocado principalmente en áreas como catalogación y descripción de especies, entre ellas: Phataria unifascialis, Luidia foliolata, Ophiocoma aethiops, Ophiocomella alexandri, Ophiothrix (Ophiothrix) rudis, Echinometra vanbrunti, Diadema mexicanum, Lanthonia longifissa, Encope micropora, Holothuria (Halodeima) kefersteinii, Holothuria (Selenkothuria) lubrica, Holothuria (Cystipus) rigida (Barraza, 1995; Barraza \& Hasbún, 2005; Carballo \& Pocasangre, 2007; Carranza \& Rivera, 2007; López-Martínez, 2017) y ecología de especies en arrecifes someros (hasta 8 $\mathrm{m}$ de profundidad) (Alvarado, Cortés, Guzman, \& Reyes-Bonilla, 2016; Segovia, Guerra \& Ramos, 2017).

También se han registrado en investigaciones enfocadas en temáticas como estructura faunística del macrobentos en esteros, en donde se menciona la presencia de algunos ofiuroideos (Amphiodia oerstedi) (Hernández \& Davis, 1979; Molina-Lara \& VargasZamora, 1994), como fauna acompañante en 
captura de larvas de camarón (Parada-Argueta \& Saez-Callejas, 1995), como parte de las descripciones de macroinvertebrados marinos que ocurren en el país (Barraza, 2000, 2001; Reyes-Bonilla \& Barraza, 2003; Barraza, 2008, 2010, 2014) y en listados de equinodermos para Centroamérica (Alvarado, Solís-Marín \& Ahearn, 2010; Alvarado \& Solís-Marín, 2013). En algunos de los estudios antes mencionados, se han realizado recolectas de organismos que han sido depositados en colecciones científicas nacionales, los cuales son fundamentales para conocer el desarrollo de la investigación con equinodermos en El Salvador; hasta la fecha se cuenta con un registro de un total de 60 especies para el país en donde las clases Holothuroidea y Ophiuroidea son las más abundantes (Segovia et al., 2017).

Sin embargo, en este contexto se desconocen con exactitud las especies de equinodermos de El Salvador depositadas en colecciones científicas nacionales y extranjeras. Por esta razón, el presente trabajo tiene como objetivo enlistar estas colecciones con sus especies, conocer los esfuerzos realizados para la recolección de estos ejemplares y evaluar el aporte de las colecciones al conocimiento del taxón en el país.

\section{MATERIALES Y MÉTODOS}

Se realizó una revisión de 18 bases de datos en línea de las principales colecciones científicas del mundo (The Natural History Museum, Londres; California Academy of Science, Museum of Comparative Zoology, National Museum of Natural History Smithsonian y Yale Peabody Museum of Natural History, E.U.A.; Charles Darwin Research Station, Ecuador; Museo Regionale di Scienze Naturali y Museo Zoologico dell'Università di Firenze, Italia; Muséum National d'Histoire Naturelle, Francia; Smithsonian Tropical Research Institute, Panamá; Museo de Zoología, Escuela de Biología, Universidad de Costa Rica, Costa Rica; Zoologisches Institut und Zoologisches Museum y Zoologische Staatssammlung München, Alemania; Zoologisk
Museum Kobenhavn, Dinamarca; Museo de Historia Natural de El Salvador, Instituto Tropical de Investigaciones Científicas e Instituto de Ciencias del Mar y Limnología, El Salvador; Instituto de Ciencias del Mar y Limnología, México), pertenecientes a museos, universidades e institutos de investigación, en donde se seleccionaron aquellas que tuvieran ejemplares de equinodermos recolectados en la costa de El Salvador, siendo las siguientes: California Academy of Sciences (CAS), National Museum of Natural History Smithsonian (NMNH), y la Colección Nacional de Equinodermos "Dra. Ma. Elena Caso" del Instituto de Ciencias del Mar y Limnología de la Universidad Nacional Autónoma de México (ICML); en caso de que alguna información tuviera restricciones, fue solicitada a los encargados de colecciones o curadores. Además, se revisaron taxonómicamente los ejemplares depositados en colecciones científicas nacionales: Instituto Tropical de Investigaciones Científicas (ITIC) (ahora Escuela de Biología), Instituto de Ciencias del Mar y Limnología (ICMARES), ambas de la Universidad de El Salvador. Se solicitó información sobre los especímenes depositados en el Museo de Historia Natural de El Salvador (MUHNES) del Ministerio de Cultura. Para formular el registro de cada ejemplar, se compiló la siguiente información: colección científica, especie recolectada, número de ejemplares, sitio (Departamento), año de recolecta y recolector.

El análisis de la información se ordenó por el listado taxonómico de las especies registradas y por medio de gráficos que representan el número de especies de equinodermos por clase registrada en cada colección, años y sitios de recolecta de ejemplares.

\section{RESULTADOS}

Los equinodermos de El Salvador se encuentran depositados en seis colecciones científicas nacionales y extranjeras, representados por 536 ejemplares que corresponden a 192 registros clasificados en 40 especies, 32 géneros y 24 familias de este grupo. Del total 
de registros, 178 se encontraron identificados hasta especie, 11 a nivel de género y tres en familia; de ellas, se registró una nueva especie de ofiuroideo para El Salvador: Ophionereis perplexa. Las colecciones científicas con mayor número de ejemplares fueron NMNH con 201 e ICMARES con 127, CAS (106), ITIC (84), ICML (12) y MUHNES (seis)
(Tabla 1). La clase con mayor representatividad de ejemplares catalogados hasta especie o género fue Ophiuroidea (13 especies y dos géneros), seguido por Holothuroidea (12 especies y tres géneros), Echinoidea (11 especies y cuatro géneros) y Asteroidea (cuatro especies y dos géneros).

TABLA 1

Listado taxonómico de los equinodermos de El Salvador depositados en colecciones científicas de museos y universidades. Ver acrónimos en la sección Materiales y Métodos

TABLE 1

Taxonomic list of echinoderms from El Salvador deposited at scientific collections of museums and universities. See acronyms in Materials and Methods section

\section{Clase Asteroidea}

Familia Astropectinidae

Astropecten sp.

Familia Luidiidae

Luidia sp.

Luidia foliolata Grube, 1866

Luidia (Platasterias) latiradiata (Gray, 1871)

Familia Ophidiasteridae

Phataria unifascialis (Gray, 1840)

Familia Oreasteridae

Nidorellia armata (Gray, 1840)

\section{Clase Ophiuroidea}

Familia Amphiuridae

Amphiodia oerstedi (Lütken, 1856)

Microphiopholis platydisca (Nielsen, 1932)

Ophiophragmus sp.

Familia Ophiotrichidae

Ophiothela mirabilis Verrill, 1867

Ophiothrix (Ophiothrix) rudis Lyman, 1874

Ophiothrix (Ophiothrix) spiculata Le Conte, 1851

Familia Ophiocomidae

Ophiocoma aethiops Lütken, 1859

Ophiocomella alexandri (Lyman, 1860)

Familia Ophiuridae

Familia Ophionereididae

Ophionereis sp.

Ophionereis annulata (Le Conte, 1851)

*Ophionereis perplexa Ziesenhenne, 19401

Familia Ophiactidae

Ophiactis savignyi (Müller \& Troschel, 1842)

Familia Ophiodermatidae

Ophioderma panamense Lütken, 1859

Ophioderma teres (Lyman, 1860)

\section{CAS NMNH ICML ICMARES MUHNES ITIC}

$\begin{array}{lll}X & X & \\ X & X & X \\ X & X & \\ & X & \\ & & \\ X & X & \end{array}$

$\mathrm{X}$

$\mathrm{X}$

$\mathrm{X}$

$\mathrm{X}$

$\begin{array}{cc}\mathrm{X} & \mathrm{X} \\ & \mathrm{X} \\ & \mathrm{X} \\ \mathrm{X} & \mathrm{X}\end{array}$

$\mathrm{X}$

$\mathrm{X}$

$\mathrm{X}$

$\mathrm{X}$

X

X $\mathrm{X}$

$\mathrm{X}$ 
TABLA 1 (Continuación) / TABLE 1 (Continued)

\begin{tabular}{|c|c|c|c|c|c|c|}
\hline & CAS & $\mathrm{NMNH}$ & ICML & ICMARES & MUHNES & ITIC \\
\hline \multicolumn{7}{|l|}{ Familia Ophiolepididae } \\
\hline Ophiolepis variegata Lütken, 1856 & & $\mathrm{X}$ & & & & \\
\hline \multicolumn{7}{|l|}{ Clase Echinoidea } \\
\hline \multicolumn{7}{|l|}{ Familia Arbaciidae } \\
\hline Arbacia stellata (Blainville, 1825; ?Gmelin, 1791) & $\mathrm{X}$ & $\mathrm{X}$ & & & & \\
\hline \multicolumn{7}{|l|}{ Familia Cidaridae } \\
\hline Eucidaris thouarsii (L. Agassiz \& Desor, 1846) & & $\mathrm{X}$ & & & & \\
\hline \multicolumn{7}{|l|}{ Familia Diadematidae } \\
\hline Astropyga pulvinata (Lamarck, 1816) & & $\mathrm{X}$ & & & & \\
\hline Diadema mexicanum A. Agassiz, 1863 & & & & $\mathrm{X}$ & & $\mathrm{X}$ \\
\hline \multicolumn{7}{|l|}{ Familia Echinometridae } \\
\hline Echinometra sp. & & & & & $\mathrm{X}$ & \\
\hline Echinometra vanbrunti A. Agassiz, 1863 & $\mathrm{X}$ & $\mathrm{X}$ & & $\mathrm{X}$ & & $\mathrm{X}$ \\
\hline Familia Mellitidae & & & & & $\mathrm{X}$ & \\
\hline Encope sp. & & & & & $\mathrm{X}$ & \\
\hline Encope grandis L. Agassiz, 1841 & & & & & $\mathrm{X}$ & \\
\hline Encope micropora L. Agassiz, 1841 & & $\mathrm{X}$ & & & & \\
\hline Lanthonia longifissa (Michelin, 1858) & $\mathrm{X}$ & $\mathrm{X}$ & $\mathrm{X}$ & & & $\mathrm{X}$ \\
\hline Mellita sp. & & & & & $\mathrm{X}$ & \\
\hline Mellita notabilis H.L. Clark, 1947 & $\mathrm{X}$ & $\mathrm{X}$ & $\mathrm{X}$ & & & \\
\hline Mellitella sp. & & $\mathrm{X}$ & & & & \\
\hline Melitella stokesii (L. Agassiz, 1841) & & $\mathrm{X}$ & & $\mathrm{X}$ & & \\
\hline \multicolumn{7}{|l|}{ Familia Prenasteridae } \\
\hline Agassizia scrobiculata Valenciennes, 1846 & $\mathrm{X}$ & $\mathrm{X}$ & & & & \\
\hline \multicolumn{7}{|l|}{ Clase Holothuroidea } \\
\hline \multicolumn{7}{|l|}{ Familia Cucumariidae } \\
\hline Neocucumis veleronis (Deichmann, 1941) & & $\mathrm{X}$ & & & & \\
\hline Familia Holothuriidae & $\mathrm{X}$ & & & & & \\
\hline Holothuria sp. & & & & & & $\mathrm{X}$ \\
\hline Holothuria (Cystipus) rigida (Selenka, 1867) & & & $\mathrm{X}$ & & & \\
\hline Holothuria (Halodeima) inornata Semper, 1868 & & & & $\mathrm{X}$ & & $\mathrm{X}$ \\
\hline Holothuria (Halodeima) kefersteinii (Selenka, 1867) & & $\mathrm{X}$ & $\mathrm{X}$ & $\mathrm{X}$ & & $\mathrm{X}$ \\
\hline Holothuria (Selenkothuria) lubrica Selenka, 1867 & $\mathrm{X}$ & $\mathrm{X}$ & & & & \\
\hline Holothuria (Semperothuria) imitans Ludwig, 1875 & & & & $\mathrm{X}$ & & \\
\hline Labidodemas americanum Deichmann, 1938 & & & $\mathrm{X}$ & & & \\
\hline \multicolumn{7}{|l|}{ Familia Phyllophoridae } \\
\hline Pentamera sp. & & $\mathrm{X}$ & & & & \\
\hline Pentamera zacae Deichmann, 1938 & & $\mathrm{X}$ & & & & \\
\hline \multicolumn{7}{|l|}{ Familia Psolidae } \\
\hline Lissothuria ornata Verrill, 1867 & & & $\mathrm{X}$ & & & \\
\hline \multicolumn{7}{|l|}{ Familia Sclerodactylidae } \\
\hline Afrocucumis ovulum (Selenka, 1867) & & & & $\mathrm{X}$ & & \\
\hline Neothyone sp. & & $\mathrm{X}$ & & & & \\
\hline Neothyone gibbosa Deichmann, 1941 & & & $\mathrm{X}$ & & & \\
\hline \multicolumn{7}{|l|}{ Familia Stichopodidae } \\
\hline Isostichopus fuscus (Ludwig, 1875) & & & & $\mathrm{X}$ & & \\
\hline Número de ejemplares & 106 & 201 & 12 & 127 & 6 & 84 \\
\hline
\end{tabular}




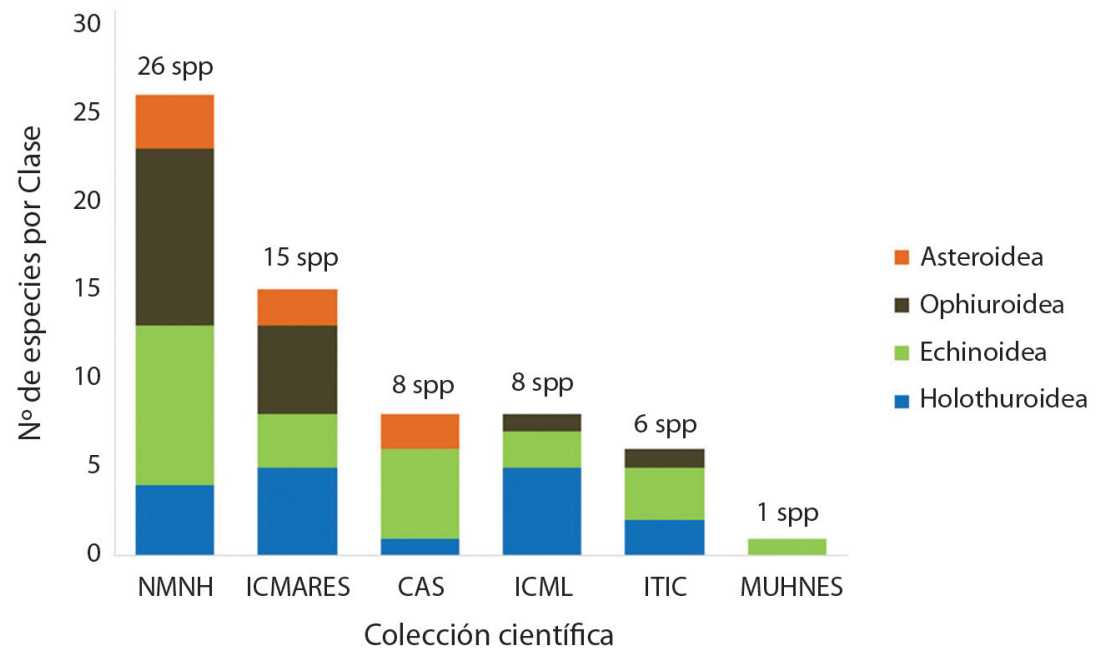

Fig. 1. Número de especies por clase depositadas en las seis colecciones científicas. Ver acrónimos en la sección Materiales y Métodos.

Fig. 1. Species numbers by class deposited in the six scientific collections. See acronyms in Materials and Methods section.

La colección que presentó mayor diversidad de especies fue el NMNH con 26, seguido por ICMARES con 15 especies agrupadas en las cuatro clases de equinodermos registradas para El Salvador (Asteroidea, Ophiuroidea, Echinoidea y Holothuroidea); seguido por CAS e ICML con ocho especies cada una, ambas con representación de tres clases (Ophiuroidea, Echinoidea y Holothuroidea); las colecciones de ITIC y MUHNES con seis y una especies, respectivamente, la primera con representación de tres clases y la segunda únicamente con registros de la clase Echinoidea (Fig. 1).

El NMNH fue la colección que posee el registro más antiguo del taxón en el país con la recolecta de un ejemplar en 1952, luego esta colección junto con la de CAS, mostraron mayor apogeo en la recolección de ejemplares durante los 70's y finalizando en la década de los 2000. El ICMARES inició la recolección en la década del 2010, exactamente desde el 2012, con mayor esfuerzo en la captura de equinodermos entre 2014 y 2015. El ITIC posee registros de ejemplares recolectados principalmente en la década de 1990 y posteriormente hasta el 2009. El MUHNES posee ejemplares recolectados en 2001. El ICML registra únicamente las especies y sitios de recolecta de los ejemplares. También cuatro (NMNH, ICMARES, ITIC e ICML) de las seis colecciones poseen algunos registros sin años de recolecta (Fig. 2).

Las recolectas se han realizado en las costas de siete departamentos del país que son La Unión, Sonsonate, Usulután, La Libertad, Ahuachapán, La Paz y San Miguel; otros registros no poseen sitio de recolecta. Todas las colecciones poseen ejemplares provenientes de La Unión, y el MUHNES como único sitio de recolección, el NMNH, ICMARES y CAS son los que presentan más registros en este departamento, 33, 24 y 19, respectivamente; Sonsonate con registros de recolectas realizadas en su mayoría por ICMARES y CAS, y en menor proporción el NMNH, ITIC e ICML; Usulután con mayores esfuerzos de recolectas realizados por el ICMARES y NMNH, en segundo lugar las colecciones de CAS (dos registros) e ICML (un registro); el ITIC posee mayor cantidad de registros en La Libertad (ocho), seguido por el NMHN e ICML; los departamentos de Ahuachapán, La Paz y San Miguel presentaron los registros más bajos en recolecta de equinodermos, sin embargo NMNH, ICMARES e ITIC poseen ejemplares de este sitio (Fig. 3). 


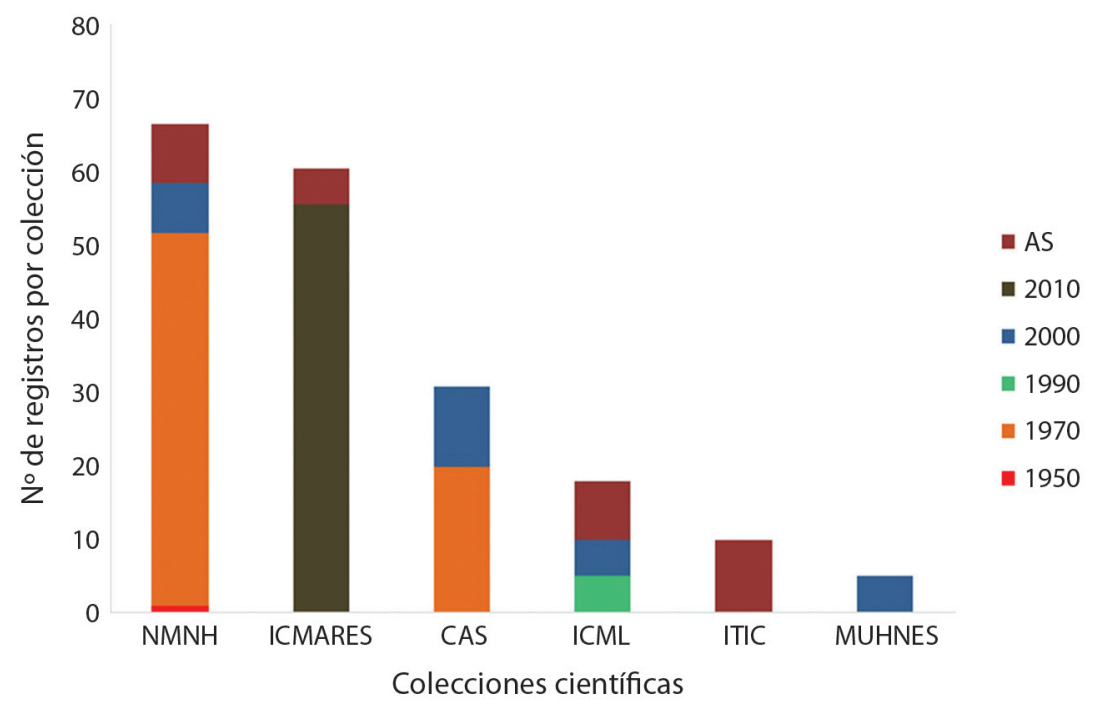

Fig. 2. Número de ejemplares recolectados por institución. SA = Sin Año. Ver acrónimos en la sección Materiales y Métodos.

Fig. 2. Specimen numbers collected by institution. SA = No Year. See acronyms in Materials and Methods section.

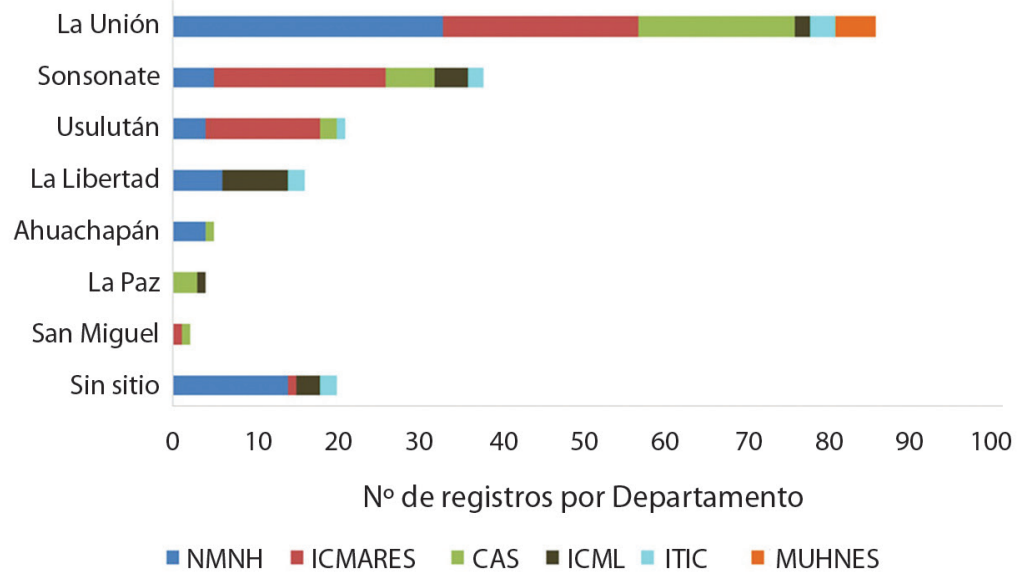

Fig. 3. Departamentos de recolecta de equinodermos en la costa de El Salvador. Ver acrónimos en la sección Materiales y Métodos.

Fig. 3. Collection Departments of echinoderms in the coast of El Salvador. See acronyms in Materials and Methods section.

\section{DISCUSIÓN}

A nivel mundial existe un total de 536 ejemplares de equinodermos recolectados en El Salvador, depositados en seis colecciones científicas, tres nacionales y tres extranjeras. La colección científica que posee mayor número de ejemplares a nivel mundial es el NMHN y a nivel nacional la del ICMARES que como explican Segovia et al. (2016) en el avance preliminar del registro de biodiversidad de equinodermos en esta colección, hasta ese momento se encontraron 98 ejemplares clasificados en once especies. Sin embargo, en este 
estudio, esta cantidad aumentó con 23 ejemplares y cuatro especies, esto es debido a los esfuerzos del Programa Nacional de Monitoreo en Arrecifes Rocosos y de Coral realizados por el ICMARES. En poco tiempo esta colección ha realizado un progreso significativo en resguardar la biodiversidad de equinodermos siendo invaluable para el país.

En cuanto a la diversidad, se registró un total 40 especies de equinodermos, entre ellas un nuevo registro Ophionereis perplexa, que representan el $66.7 \%$ del total reportado para El Salvador (Barraza \& Hasbún, 2005; Carranza \& Rivera, 2007; Alvarado et al., 2010; Alvarado \& Solís-Marín, 2013), es decir que el $33.3 \%$ aún no se encuentra depositado en colecciones científicas pero si descrito en la literatura antes citada, por lo que la recolecta de ejemplares debe ser enfocada en estas especies. Es importante resaltar que los registros enlistados en este estudio pertenecen, en su mayoría, a equinodermos de zonas someras (profundidades hasta $30 \mathrm{~m}$ ), lo que indica que los esfuerzos de recolección de ejemplares deben enfocarse en aguas profundas.

De acuerdo con los autores antes mencionados, la riqueza de equinodermos por clase en El Salvador se compone de la siguiente forma: Holothuroidea (19 especies), seguido por Ophiuroidea (18 especies), Echinoidea con (11 especies) y Asteroidea con (ocho especies). En el presente estudio se registró mayor diversidad de especies de la clase Ophiuroidea depositadas en las colecciones científicas y extranjeras, de la cual se encontraron 13 especies que representan el $72.2 \%$ de este depósito, en esta misma forma para las clases restantes: Holothuroidea (63\%), Echinoidea (73\%) y Asteroidea (50\%).

El nuevo registro del ofiuroideo Ophionereis perplexa supone un llenado en la distribución de esta especie, registrada para el Pacífico mexicano e islas Galápagos (Maluf, 1991; Solís-Marín, Laguarda-Figueras, DuránGonzález, Ahearn, \& Torres-Vega, 2005; Granja-Fernández, Herrero-Pérezrul, López-Pérez, Hernández-Morales, \& Rangel-Solís, 2015) y el aumento de la biodiversidad de equinodermos para El Salvador.

Los ejemplares depositados en el NMNH y CAS corresponden a dos esfuerzos: el primero realizado por las expediciones científicas de los Cuerpos de Paz de Estados Unidos, durante la década de 1970, Echeverría (2013) describe que estos fueron iniciativa del entonces Servicio Nacional de Parques y Vida Silvestre (PANAVIS) y el MUHNES con el objetivo de conocer la biodiversidad de El Salvador. Por esta razón, las recolectas de equinodermos provienen de las costas de La Unión, Sonsonate, Usulután y La Libertad. El segundo esfuerzo realizado por el buque de investigación URRACÁ del STRI que en coordinación con el Ministerio de Medio Ambiente muestrearon los Departamentos de Sonsonate, La Unión y Usulután, recolectaron equinodermos durante el 2001, cinco de ellos fueron depositados en la colección del MUHNES (Barraza, 2001; Barraza \& Hasbún, 2005) y el resto en NMNH y CAS.

En cuanto a los ejemplares depositados en el ITIC que datan de la década de los 90's, corresponden a recolectas realizadas durante visitas de campo de las cátedras de la Escuela de Biología (Chicas-Batres \& González-Leiva, 2015) en Sonsonate, La Libertad, La Paz y San Miguel; en 2009 se registraron cinco recolectas realizadas por Juan José Alvarado durante su investigación sobre la bioerosión causada por D. mexicanum en los arrecifes del Pacífico Oriental Tropical (Alvarado et al. 2016), recolectas provenientes de Sonsonate y La Libertad.

Por otra parte, los ejemplares depositados en el ICML provienen de recolectas realizadas por Carlos Roberto Hasbún, las cuales provienen de La Unión, Sonsonate, La Libertad y Usulután. En esta colección se registraron dos especies importantes: una de ellas es $H$. (Cystipus) rigida, especie registrada por primera en vez en El Salvador por Carranza y Rivera (2007) quienes describen que los individuos se encontraron en el manglar de la Bahía de Jiquilisco entre raíces de Rhizophora mangle, entre 17-22 $\mathrm{cm}$ de profundidad dentro del sustrato fangoso, asociado a bivalvos como Anadara tuberculosa y Anadara similis. La 
otra es Ophionereis perplexa recolectado en Maculís, La Unión. Cabe resaltar que son los únicos ejemplares de estas especies registrados en todas las colecciones científicas presentadas en este estudio.

En cuanto a las recolectas del ICMARES, corresponden al esfuerzo del Programa Nacional de Monitoreo en Arrecifes Rocosos y de Coral, que según Segovia et al. (2016), los esfuerzos de muestreo con equinodermos se han realizado en seis localidades: Puerto de Acajutla, Área Natural Protegida Los Cóbanos (Sonsonate), La Libertad, Bahía de Jiquilisco (Usulután), Bahía de La Unión y Punta Amapala (La Unión) con recolección de organismos entre 2012-2018. La mayoría de las especies recolectadas por el instituto corresponden a equinodermos de arrecifes rocosos y coralinos a profundidades menores de $3 \mathrm{~m}$, entre ellas $O$. alexandri, $O$. aethiops, $O$. (Ophiothrix) rudis, E. vanbrunti, D. mexicanum, H. (Halodeima) kefersteinii y Afrocucumis ovulum (Segovia et al., 2017), por lo que la abundancia de estas especies dentro de esta colección es mayor.

En términos generales, la representación de ejemplares en colecciones científicas nacionales y extranjeras es aceptable; sin embargo, en El Salvador el conocimiento sobre equinodermos debe reforzarse. Esto se refleja en las investigaciones limitadas con el taxón y que se puede traducir en una cantidad moderada de especies y ejemplares representadas en las colecciones, tomando en cuenta que estas desarrollan un papel valioso como respaldo físico de la diversidad de equinodermos que posee El Salvador y que aún es poco conocida. En relación con esto y para resaltar la importancia de las colecciones científicas, en el presente estudio se encontró depositado en la colección del ICML, el ofiuroideo Ophionereis perplexa, aumentando la biodiversidad de equinodermos a 61 especies reportadas para El Salvador.

Por lo tanto, deben continuar los esfuerzos en la identificación y catalogación de especies de aguas someras e iniciar las investigaciones con el taxón en aguas profundas en los sitios donde se han realizado más recolectas de equinodermos como Sonsonate, La Unión, Usulután y La Libertad e investigar los poco estudiados como Ahuachapán, La Paz y San Miguel.

Declaración de ética: los autores declaran que todos están de acuerdo con esta publicación y que han hecho aportes que justifican su autoría; que no hay conflicto de interés de ningún tipo; y que han cumplido con todos los requisitos y procedimientos éticos y legales pertinentes. Todas las fuentes de financiamiento se detallan plena y claramente en la sección de agradecimientos. El respectivo documento legal firmado se encuentra en los archivos de la revista.

\section{AGRADECIMIENTOS}

A D. Durán y M. Salinas, por proveernos información sobre las colecciones de la Escuela de Biología de la Universidad de El Salvador. A E. Echeverría por brindarnos información sobre los ejemplares de equinodermos presentes en el Museo de Historia de Natural de El Salvador. A E. Barraza por proporcionarnos algunas de las primeras investigaciones con equinodermos en el país. R. Vargas y J.J. Alvarado por compartirnos información sobre los ejemplares de equinodermos del Museo de Zoología de la Universidad de Costa Rica. A todas las personas que con sus observaciones y comentarios aportaron a la presentación de este trabajo.

\section{RESUMEN}

Introducción: El valor de las colecciones científicas radica en que los registros están vinculados directamente a especímenes físicos permitiendo conocer los avances en taxonomía, biogeografía e historia evolutiva de las especies. En El Salvador, las colecciones científicas están representadas en su mayoría por ejemplares de vertebrados terrestres y en menor proporción invertebrados marinos, en específico para el grupo de equinodermos su representación es escasa. Objetivo: Enlistar las colecciones científicas en las cuales se encuentran depositados ejemplares de equinodermos de El Salvador, conocer los esfuerzos realizados para la recolección de estos ejemplares y evaluar el aporte de las colecciones al conocimiento del taxón en el país. Métodos: Revisión de bases de datos abiertas de 18 colecciones extranjeras y nacionales; recopilando 
información biológica, número de ejemplares, sitio, año de recolecta y recolector. Resultados: Se encontraron 536 ejemplares en 192 registros de equinodermos depositados en seis colecciones científicas, nacionales y extranjeras, clasificadas en 40 especies, una de ellas nueva para El Salvador: Ophionereis perplexa. El National Museum of Natural History Smithsonian y el Instituto de Ciencias del Mar y Limnología de la Universidad de El Salvador albergan el mayor número de ejemplares, el primero con los ejemplares más antiguos, con más recolectas en La Unión y Sonsonate para ambas colecciones. Conclusiones: La representación de ejemplares en colecciones científicas nacionales y extranjeras es aceptable, registrando en los depósitos el $66.7 \%$ del total reportado para El Salvador. Se recomienda continuar los esfuerzos en la identificación y catalogación de especies de aguas someras e iniciar las investigaciones con el taxón en aguas profundas.

Palabras clave: recolectas; biodiversidad; Ophionereis perplexa; registros; La Unión.

\section{REFERENCIAS}

Alvarado, J.J., Solís-Marín, F.A., \& Ahearn, G.C. (2010). Echinoderm (Echinodermata) diversity in the Pacific coast of Central America. Marine Biodiversity, 40, $45-56$.

Alvarado, J.J. \& Solís-Marín, F.A. (2013). Echinoderm Research and Diversity in Latin America. En J.J. Alvarado \& F.A. Solís-Marín (Eds.), Echinoderm Research and Diversity in Latin America (pp. 1-9). Estados Unidos: Elsevier.

Alvarado, J.J., Cortés, J., Guzman, H., Reyes-Bonilla, H. (2016). Bioerosion by the sea urchin Diadema mexicanum along Eastern Tropical Pacific coral reefs. Marine Ecology, 37(5), 1088-1102.

Barraza, J.E. (1995). Equinodermos de El Salvador. En F. Serrano (Ed.), Historia Natural y Ecología de El Salvador, Tomo II (pp. 87-99). San Salvador: Ministerio de Educación.

Barraza, J.E. (2000). Comentarios sobre la biodiversidad de invertebrados marinos de El Salvador. San Salvador, El Salvador: Publicación Ocasional No 2 . Ministerio de Medio Ambiente y Recursos Naturales.

Barraza, J.E. (2001). Informe preliminar de la expedición El Salvador (Pacifico Este Tropical), Instituto Smithsonian de Investigación Tropical, STRI, NMNH, Museo Nacional de Historia Natural, Institución Smithsonian $R / V$ URRACÁ (Washington, DC). El Salvador: Ministerio de Medio Ambiente y Recursos Naturales de El Salvador.

Barraza, J.E. (2008). Revisión sobre algunos taxa de macroinvertebrados acuáticos en El Salvador. San
Salvador, El Salvador: Ministerio de Medio Ambiente y Recursos Naturales.

Barraza, J.E. (2010). Biodiversidad de Bahía de Jiquilisco. En C.G. Rivera \& T.C. Cuéllar (Eds.), El ecosistema de manglar de la Bahía de Jiquilisco: Sector Occidental (pp. 13-32). El Salvador: FIAES.

Barraza, J.E. (2014). Invertebrados marinos de El Salvador. San Salvador, El Salvador: Ministerio de Medio Ambiente y Recursos Naturales.

Barraza, J.E., \& Hasbún, C.R. (2005). Los equinodermos (Echinodermata) de El Salvador. Revista de Biología Tropical, 53(3), 139-146.

Carballo, R.E., \& Pocasangre, X.M. (2007). Composición y estructura de la fauna intermareal de equinodermos en el Sistema Arrecifal Rocoso Los Cóbanos, Departamento de Sonsonate, El Salvador (Tesis de Licenciatura). Escuela de Biología, Universidad de El Salvador, El Salvador.

Carranza, O.A., \& Rivera, A.M. (2007). Nuevo reporte de la especie Holothuria (Cystipus) rigida para El Salvador, C.A. El Salvador: Comité de Desarrollo Empresarial y Ambiental de Puerto Parada.

Chicas-Batres, F.A. \& González-Leiva, J.A. (2015). Peces de El Salvador depositados en colección científica de museos y universidades. En L.F. Del MoralFlores, A.J. Ramírez-Villalobos, J.A. Martínez-Pérez, \& A.F. González-Acosta (Eds.), Colecciones Ictiológicas de Latinoamérica (pp. 107-116). México: UNAM-FESI-SIMAC.

Cristín, A., \& Perrilliat, M.C. (2011). Las colecciones científicas y la protección del patrimonio paleontológico. Boletín de la Sociedad Geológica Mexicana, 63(3), 421-427.

Darrigan, G. (2012). Las colecciones biológicas: ¿Para qué?. Boletín Biológica, 23, 28-31.

Echeverría, E.E. (2013). El Museo de Historia Natural de El Salvador: Inicio, evolución y futuro. El Salvador: Secretaría de Cultura de la Presidencia, Dirección de Publicaciones e impresos.

Granja-Fernández, R., Herrero-Pérezrul, M.D., LópezPérez, A., Hernández-Morales, A., \& Rangel-Solís, P.D. (2015). A literature review of the Ophiuroidea (Echinodermata) from the Pacific coast of Mexico. Revista de Biología Tropical, 63(2), 37-47.

Hernández, M.A., \& Davis, J.H. (1979). Estudio de algunos factores físico-químicos que influyen en la diversidad de las especies de la macrofauna bentónica del estuario de El Tamarindo. San Salvador, El Salvador: Museo de Historia Natural de El Salvador.

ICML-UNAM (Instituto de Ciencias del Mar y Limnología, Universidad Nacional Autónoma de México). (2019). Colección Nacional de Equinodermos. Recuperado de https://www.icmyl.unam.mx/es/quienes-somos/ colecciones/coleccion-nacional-de-equinodermos 
López-Martínez, R.E. (2017). Distribución y abundancia de la fauna intermareal de equinodermos en el Área Natural Protegida Complejo Los Cóbanos, Sonsonate, El Salvador (Tesis de Licenciatura). Escuela de Biología, Universidad de El Salvador, El Salvador.

Maluf, L.Y. (1991). Echinoderm Fauna of the Galápagos Islands. En M.J. James (Ed.), Galápagos Marine Invertebrates. Taxonomy, Biogeography, and Evolution in Darwin's Islands (pp. 345-367). Nueva YorkLondres: Plenum Press.

Molina-Lara, O.A., \& Vargas-Zamora, J.A. (1994). Estructura del macrobentos del estero de Jaltepeque, El Salvador. Revista de Biología Tropical, 42(1-2), $165-174$.

Parada-Argueta, O., \& Saez-Callejas, N. (1995). Estudio de la fauna acompañante en la captura de postlarvas de camarones peneidos en la Bahía de Jiquilisco, El Salvador. Ciudad de Panamá, Panamá: Simposio Ecosistema de Manglares en el Pacífico Centroamericano. PRADEPESCA.

Reyes-Bonilla, H., \& Barraza, J.E. (2003). Coral and associated marine communities from El Salvador. En J. Cortés (Ed.), Latin American Coral Reefs (pp. 351360). Londres: Elsevier Science.
Segovia, J., Guerra, G., López, G., \& Ramos, F. (2016). Avance preliminar del registro de biodiversidad de equinodermos (Echinodermata) de El Salvador, Centroamérica. San José, Costa Rica: Libro de Resúmenes del $3^{\circ}$ Congreso Latinoamericano de Equinodermos.

Segovia, J., Guerra, G., \& Ramos, F. (2017). Riqueza y distribución de equinodermos en los arrecifes rocosos de Punta Amapala y Los Cóbanos, El Salvador. Revista de Biología Tropical, 65(1), S92-S100.

Simmons, J.E., \& Muñoz-Saba, Y. (2005). Cuidado, manejo y conservación de las colecciones biológicas. Bogotá, Colombia: Panamericana, Formas e Impresos S.A

Solís-Marín, F.A., Laguarda-Figueras, A., Durán-González, A., Ahearn, C.G., \& Torres-Vega, J. (2005). Equinodermos (Echinodermata) del Golfo de California, México. Revista de Biología Tropical, 53(3), 123-137.

UNAM (Universidad Nacional Autónoma de México). (2013). Única en México y la mejor de Latinoamérica, la Colección Nacional de Equinodermos. Recuperado de https://www.dgcs.unam.mx/boletin/ bdboletin/2013_438.html 